\title{
IRREDUCIBLE SPACES OF MODULAR UNITS
}

\author{
DAVID E. ROHRLICH
}

Fix a prime $p \geqslant 7$, put $G=\operatorname{PSL}\left(2, \mathbb{F}_{p}\right)$, and write $U$ for the multiplicative group of modular units of level $p$. We shall determine the irreducible subspaces of the natural representation of $G$ on $U / U^{p}$. The outcome of the calculation can be described as follows: Every irreducible nontrivial representation of $G$ over $\mathbb{F}_{p}$ occurs with multiplicity one in the maximal semisimple subspace of the "noncongruence part" of $U / U^{p}$ (to be defined). Apart from the formulation and some slight differences arising from the choice of group $\left(\operatorname{PSL}\left(2, \mathbb{F}_{p}\right)\right.$ instead of $\left.\operatorname{GL}\left(2, \mathbb{F}_{p}\right) /\{ \pm 1\}\right)$, the result is already in Gross [2]. Presumably one can give conditions as in [6] and [7] which ensure that the unit group remains large after descent and specialization to a number field, but this problem will not be addressed here.

For the sake of perspective, it is useful to recall that the natural representation of $G$ on the space of modular forms of weight 2 and level $p$ was decomposed into irreducibles in two papers of Hecke [3], [4]. As one would expect, most of the work in these papers goes into decomposing the space of cusp forms, but it is actually the space of Eisenstein series - dealt with by Hecke in a few lines - which has some bearing on the present note. The reason is simple: if $f \in U$ then $(d \log f) / d z$ is an Eisenstein series of weight 2 and level $p$. In fact the space of all such Eisenstein series is simply $\mathbb{C} \otimes_{\mathbb{Z}}(d \log U) / d z$. Furthermore, since the kernel of $f \mapsto(d \log f) / d z$ is the subgroup of constant functions $\mathbb{C}^{\times} \subset U^{p}$, we see that $U / U^{p}$ is isomorphic as an $\mathbb{F}_{p}[G]$-module to $\mathbb{F}_{p} \otimes_{\mathbb{Z}}(d \log U) / d z$. Thus the representation of $G$ on $U / U^{p}$ arises via tensor product with $\mathbb{F}_{p}$ from a $G$-stable $\mathbb{Z}$-form of the space of Eisenstein series. It follows that the semisimplification of $U / U^{p}$ can be computed directly from Hecke's decomposition of the space of Eisenstein series into irreducibles.

But the structure of $U / U^{p}$ itself is another matter. To determine whether a given irreducible constituent of $U / U^{p}$ actually occurs as a subspace we must turn to the work of Kubert and Lang [5], which reduces the problem to an elementary exercise. The present note is nothing more than a solution to the exercise: but however trite, it is nonetheless a heartfelt acknowledgment of an enormous personal debt to Serge Lang. I would also like to acknowledge the help provided by the referee of [7], whose suggestion for simplifying the proof of Proposition 7 of [7] turned out to be an essential ingredient of the present work.

\section{The Module of Parameters}

The $\mathbb{Z}[G]$-module $M$ introduced below is a first approximation to the domain of the Kubert-Lang map parametrizing $U$. Our goal is to decompose the associated representation of $G$ on the vector space $V=M / p M$ over $\mathbb{F}_{p}$.

1.1. Preliminaries. The irreducible representations of $G$ in characteristic $p$ can be classified using a single invariant: their dimension. Indeed for each integer $k$ satisfying $0 \leqslant k \leqslant(p-1) / 2$ there is an absolutely irreducible representation $\sigma_{k}$ of $G$ over $\mathbb{F}_{p}$ of dimension $2 k+1$, and $\sigma_{k}$ is unique up to isomorphism. Furthermore 
every irreducible representations of $G$ in characteristic $p$ is isomorphic to some $\sigma_{k}$. In order to work with an explicit model we shall take $\sigma_{k}$ to be the $(2 k)$ th symmetric power of the tautological two-dimensional projective representation of $G$. Then the space of $\sigma_{k}$ consists of binary homogeneous polynomials $f(x, y)$ of degree $2 k$ over $\mathbb{F}_{p}$, and the action of $G$ is given by the formula

$$
\left(\sigma_{k}(g) f\right)(x, y)=f(a x+c y, b x+d y),
$$

where $g$ is the image in $G$ of the element

$$
\tilde{g}=\left(\begin{array}{ll}
a & b \\
c & d
\end{array}\right)
$$

of $\operatorname{SL}\left(2, \mathbb{F}_{p}\right)$.

Put $R=\mathbb{F}_{p}^{2} \backslash\{(0,0)\}$. We define $M$ to be the free $\mathbb{Z}$-module of rank $\left(p^{2}-1\right) / 2$ consisting of functions $m: R \rightarrow \mathbb{Z}$ such that $m(-r)=m(r)$ for $r \in R$. An action of $G$ on $M$ is given by the formula

$$
(g \cdot m)(r)=m(r \tilde{g})
$$

where $\tilde{g}$ is either of the two lifts of $g$ to $\operatorname{SL}\left(2, \mathbb{F}_{p}\right)$ and $r \tilde{g}$ is the product of the $1 \times 2$ row vector $r$ and the matrix $\tilde{g}$. Of course this action is formally the same as (1), except that $m$ is now an even function $R \rightarrow \mathbb{Z}$ rather than a homogeneous polynomial over $\mathbb{F}_{p}$.

Given a field $F$, put $V_{F}=F \otimes_{\mathbb{Z}} M$ and extend the action (3) by linearity to a representation $\tau_{F}$ of $G$ on $V_{F}$. We can identify $V_{F}$ with the vector space of dimension $\left(p^{2}-1\right) / 2$ over $F$ consisting of even functions $m: R \rightarrow F$, and then the action of $G$ is again formally the same as in (1) and (3). We are primarily interested in the case $F=\mathbb{F}_{p}$, and in this case we write $V_{F}$ and $\tau_{F}$ simply as $V$ and $\tau$.

1.2. Irreducible constituents. Write $B$ for the image in $G$ of the upper triangular subgroup of $\mathrm{SL}\left(2, \mathbb{F}_{p}\right)$ and $N \subset B$ for the image of the strictly upper triangular subgroup (i. e. the subgroup defined by the conditions $c=0, a=d=1$ in (2)). We denote the trivial one-dimensional character of any group by 1 , leaving both the group and the implicit field of scalars to be inferred from context. In the following proposition, for example, 1 is the trivial one-dimensional character of $N$ with values in $F$, and $\operatorname{ind}_{N}^{G} 1$ is the representation of $G$ over $F$ which it induces.

Proposition 1. $\tau_{F} \cong \operatorname{ind}_{N}^{G} 1$.

Proof. Take the space of $\operatorname{ind}_{N}^{G} 1$ to consist of functions $f: G \rightarrow F$ satisfying $f(n g)=$ $f(g)$ for $n \in N$ and $g \in G$, with $G$ acting by right translation. As we have already noted, $V_{F}$ is also a space of functions, namely the space of even functions $m: R \rightarrow F$. Furthermore, given $f$ in the space of $\operatorname{ind}_{N}^{G} 1$ we obtain an element $m_{f} \in V_{F}$ by setting $m_{f}(r)=f(g)$ if $e \tilde{g}= \pm r$, where $e$ is the row vector $(0,1) \in R$. The map $f \mapsto m_{f}$ is redily verified to be $G$-equivariant and injective, and its domain and range both have dimension $\left(p^{2}-1\right) / 2$.

We now take $F=\mathbb{F}_{p}$ and compute the semisimplification of $\tau$ :

Proposition 2. The multiplicity of $\sigma_{k}$ as a constituent of $\tau$ is 1 if $k=0$ or $k=(p-1) / 2$ and 2 if $1 \leqslant k \leqslant(p-3) / 2$.

Proof. Given $t \in \mathbb{F}_{p}^{\times}$, let $a(t)$ denote the image in $B$ of the diagonal matrix with diagonal entries $t, t^{-1}$. The map $t \mapsto a(t)$ induces an isomorphism of quotient 
groups $\mathbb{F}_{p}^{\times} /\{ \pm 1\} \cong B / N$, and we can compose the inverse of this isomorphism with even powers of the Teichmüller character $\omega: \mathbb{F}_{p}^{\times} \rightarrow \mathbb{Z}_{p}^{\times}$to obtain characters of $B$. More precisely, we define $\xi_{k}: B \rightarrow \mathbb{Q}_{p}^{\times}(0 \leqslant k \leqslant(p-3) / 2)$ by setting

$$
\xi_{k}(a(t) n)=\omega(t)^{2 k} \quad\left(t \in \mathbb{F}_{p}^{\times}, n \in N\right) .
$$

Then $\operatorname{ind}_{N}^{B} 1 \cong \oplus_{k=0}^{(p-3) / 2} \xi_{k}$, whence Proposition 1 and the identification $\operatorname{ind}_{N}^{G} 1=$ $\operatorname{ind}_{B}^{G}\left(\operatorname{ind}_{N}^{B} 1\right)$ give

$$
\tau_{\mathbb{Q}_{p}} \cong \oplus_{k=0}^{(p-3) / 2} \pi_{k}
$$

with $\pi_{k}=\operatorname{ind}_{B}^{G} \xi_{k}$ (cf. formula (22) of [3]). We remark that $\pi_{0} \cong 1 \oplus \eta$ with an absolutely irreducible representation $\eta$ of dimension $p$ over $\mathbb{Q}_{p}$, while if $p \equiv 1 \bmod 4$ then $\pi_{(p-1) / 4}$ decomposes over $\overline{\mathbb{Q}}_{p}$ as the direct sum of two inequivalent irreducible representations $\zeta$ and $\zeta^{\prime}$ of dimension $(p+1) / 2$. Apart from these exceptions, the direct summands in (4) are asbolutely irreducible (although not distinct, as $\pi_{k} \cong \pi_{(p-1-2 k) / 2}$ for $\left.1 \leqslant k \leqslant(p-3) / 2\right)$.

Put $\mathcal{M}=\mathbb{Z}_{p} \otimes_{\mathbb{Z}} M$. Then $\mathcal{M}$ is a $G$-stable $\mathbb{Z}_{p}$-lattice in $V_{\mathbb{Q}_{p}}$ and $V=\mathbb{F}_{p} \otimes \mathcal{M}$. Hence the semisimplification of $V$ can be read from (4) and the mod- $p$ decomposition numbers of $G$. These decomposition numbers are implicit in Brauer-Nesbitt [1] (p. 590) and explicitly computed in Srinivasan [8] (pp. $107-108$ ). In applying [8], note that for $n=1$ her $\Phi\left(r_{0}\right)$ and $\varphi\left(r_{0}\right)$ coincide. Hence taking $r_{0}=2 k$ in formula (3.5) of [8], we find that the character of our $\pi_{k}$ coincides on $p$-regular conjugacy classes with the sum of the Brauer characters of our $\sigma_{k}$ and $\sigma_{(p-1-2 k) / 2}$. In the first instance this conclusion holds only when $1 \leqslant k \leqslant(p-3) / 2$ and $k \neq(p-1) / 4$, but in fact it holds also when $k=0$ (by the first three lines on p. 108 of [8]) and when $k=(p-1) / 4$ (by formula (3.7) of [8]). The upshot is that in all cases, the semisimplification of the reduction modulo $p$ of $\pi_{k}$ coincides with $\sigma_{k} \oplus \sigma_{(p-1-2 k) / 2}$. Hence the proposition follows from (4).

1.3. Irreducible subspaces and quotient spaces. Next we determine the multiplicity of $\sigma_{k}$ as a quotient representation of $\tau$. Given representations $\alpha$ and $\beta$ of a group $H$ on vector spaces $W_{\alpha}$ and $W_{\beta}$ over a field $F$, write $\operatorname{Hom}_{F[H]}(\alpha, \beta)$ for $\operatorname{Hom}_{F[H]}\left(W_{\alpha}, W_{\beta}\right)$.

Proposition 3. For $0 \leqslant k \leqslant(p-1) / 2$,

$$
\operatorname{dim}_{\mathbb{F}_{p}} \operatorname{Hom}_{\mathbb{F}_{p}[G]}\left(\tau, \sigma_{k}\right)=1 .
$$

Proof. Proposition 1 and Frobenius reciprocity give

$$
\operatorname{Hom}_{\mathbb{F}_{p}[G]}\left(\tau, \sigma_{k}\right) \cong \operatorname{Hom}_{\mathbb{F}_{p}[N]}\left(1, \operatorname{res}_{N}^{G} \sigma_{k}\right) \text {. }
$$

Now $N$ is generated by the element $u$ corresponding to the choices $a=b=d=1$ and $c=0$ in (2), so it suffices to see that the subspace of vectors fixed by $\sigma_{k}(u)$ is one-dimensional. Let $A$ be the matrix of $\sigma_{k}(u)$ relative to the ordered basis $x^{2 k}, x^{2 k-1} y, \ldots, y^{2 k}$, and let $a_{i j}$ be the $(i, j)$-entry of $A$ for $1 \leqslant i, j \leqslant 2 k+1$. Using (1) to write $\left(\sigma_{k}(u) f\right)(x, y)=f(x, x+y)$, one readily verifies that $A$ is upper triangular, that $a_{i i}=1$ for all $i$, and that $a_{i, i+1} \neq 0$ for $1 \leqslant i \leqslant k$. It follows that the Jordan normal form of $A$ consists of a single Jordan block, whence $x^{2 k}$ is the unique eigenvector of $\sigma_{k}(u)$ up to scalar multiples.

A similar statement holds for subrepresentations: 
Proposition 4. For $0 \leqslant k \leqslant(p-1) / 2$,

$$
\operatorname{dim}_{\mathbb{F}_{p}} \operatorname{Hom}_{\mathbb{F}_{p}[G]}\left(\sigma_{k}, \tau\right)=1 .
$$

Proof. In view of Proposition 3 it suffices to see that both $\sigma_{k}$ and $\tau$ are self-dual. The self-duality of $\sigma_{k}$ follows from the fact that irreducible representations of $G$ over $\mathbb{F}_{p}$ are determined up to isomorphism by their dimension. The self-duality of $\tau$ follows from the fact that the symmetric bilinear form

$$
\left\langle m, m^{\prime}\right\rangle=\sum_{r \in R^{\prime}} m(r) m^{\prime}(r) \quad\left(m, m^{\prime} \in V\right)
$$

is nondegenerate and $G$-invariant.

1.4. Homogeneous components. Recall that $\mathcal{M}=\mathbb{Z}_{p} \otimes_{\mathbb{Z}} M$ and that $\omega: \mathbb{F}_{p}^{\times} \rightarrow \mathbb{Z}_{p}^{\times}$ is the Teichmüller character. We shall view the elements of $\mathcal{M}$ as even functions $m: R \rightarrow \mathbb{Z}_{p}$. We define $\mathcal{M}^{(k)}$ to be the $\mathbb{Z}_{p}$-submodule of $\mathcal{M}$ consisting of those $m$ such that

$$
m(t r)=\omega(t)^{2 k} m(r)
$$

for $t \in \mathbb{F}_{p}^{\times}$and $r=\left(r_{1}, r_{2}\right) \in R$, where $t r=\left(t r_{1}, t r_{2}\right)$. The linear operators $e^{(k)}: \mathcal{M} \rightarrow \mathcal{M}$ given by

$$
\left(e^{(k)} m\right)(r)=\frac{1}{p-1} \sum_{t \in \mathbb{F}_{p}^{\times}} \omega^{-(k)}(t) m(t r)
$$

$(0 \leqslant k \leqslant(p-3) / 2)$ form a family of orthogonal idempotents projecting $\mathcal{M}$ onto the respective submodules $\mathcal{M}^{(k)}$ and summing to the identity, so we have

$$
\mathcal{M}=\oplus_{k=0}^{(p-3) / 2} \mathcal{M}^{(k)} .
$$

In fact (7) is a decomposition into $\mathbb{Z}_{p}[G]$-submodules, because the idempotents $e^{(k)}$ commute with the action of $G$. Hence the space of $\tau$ likewise decomposes into $G$-stable subspaces:

$$
V=\oplus_{k=0}^{(p-3) / 2} V^{(k)}
$$

with $V^{(k)}=\mathbb{F}_{p} \otimes_{\mathbb{Z}_{p}} \mathcal{M}^{(k)}$. Let $\tau^{(k)}$ denote the representation of $G$ on $V^{(k)}$.

Proposition 5. If $1 \leqslant k \leqslant(p-3) / 2$ then $\tau^{(k)}$ has a unique irreducible subrepresentation and a unique irreducible quotient representation, and they are equivalent to $\sigma_{k}$ and $\sigma_{(p-1-2 k) / 2}$ respectively. On the other hand, $\tau^{(0)} \cong \sigma_{0} \oplus \sigma_{(p-1) / 2}$.

Proof. The first point is that the free $\mathbb{Z}_{p}$-module $\mathcal{M}^{(k)}$ has rank $p+1$. Indeed for each of the $p+1$ lines $\ell$ through the origin in $\mathbb{F}_{p}^{2}$, fix an element $r_{\ell} \in R$ which spans $\ell$, and define a function $f_{\ell, k} \in \mathcal{M}^{(k)}$ by

$$
f_{\ell, k}(r)= \begin{cases}\omega(t)^{2 k} & \text { if } r=t r_{\ell} \text { with } t \in \mathbb{F}_{p}^{\times} \\ 0 & \text { if } r \notin \ell .\end{cases}
$$

For fixed $k$ the $p+1$ functions $f_{\ell, k}$ have pairwise disjoint supports and are therefore linearly independent over $\mathbb{Z}_{p}$. Hence $\mathcal{M}^{(k)}$ has rank at least $p+1$. But $\mathcal{M}$ has rank $(p+1)(p-1) / 2$, so we deduce from $(7)$ that $\mathcal{M}^{(k)}$ has rank exactly $p+1$, as claimed.

It follows that $V^{(k)}$ has dimension $p+1$ over $\mathbb{F}_{p}$. But an irreducible representation of $G$ over $\mathbb{F}_{p}$ has dimension $\leqslant p$, so $V^{(k)}$ has a proper irreducible subspace and 
hence at least two irreducible constituents. On the other hand, $V$ has exactly $p-1$ irreducible constituents (Proposition 2), so we deduce from (8) that $V^{(k)}$ has exactly two constituents.

To identify these constituents up to isomorphism, we introduce a $\mathbb{Z}[G]$-submodule $\mathcal{N}_{k}$ of $\mathcal{M}$ for $0 \leqslant k \leqslant(p-3) / 2$. Given $m \in \mathcal{M}$, let $\bar{m}: R \rightarrow \mathbb{F}_{p}$ denote the reduction of $m$ modulo $p$. We define $\mathcal{N}_{k} \subset \mathcal{M}$ to be the submodule consisting of all $m$ such that $\bar{m}$ coincides with a binary homogeneous polynomial of degree $2 k$ over $\mathbb{F}_{p}$. Strictly speaking, we should say "coincides with the function $R \rightarrow \mathbb{F}_{p}$ defined by" such a polynomial, but the distinction is moot: a homogeneous polynomial of degree $<p$ which vanishes on $R$ is zero. Thus the map $m \mapsto \bar{m}$ determines an embedding of $\mathcal{N}_{k} /\left(\mathcal{N}_{k} \cap p \mathcal{M}\right)$ into the space of $\sigma_{k}$. In fact this embedding is surjective and hence a $G$-isomorphism, because any even function $R \rightarrow \mathbb{F}_{p}$ can be lifted to an even function $R \rightarrow \mathbb{Z}_{p}$.

Now put $\mathcal{N}_{k}^{(l)}=e^{(l)} \mathcal{N}_{k}(0 \leqslant l \leqslant(p-3) / 2)$. It is readily verified that if $l \neq k$ then the image of $\mathcal{N}_{k}^{(l)}$ under $m \mapsto \bar{m}$ is $\{0\}$. On the other hand, we have just seen that the map $m \mapsto \bar{m}$ gives a $G$-isomorphism of $\mathcal{N}_{k} /\left(\mathcal{N}_{k} \cap p \mathcal{M}\right)$ onto the space of $\sigma_{k}$. It follows that the domain of this $G$-isomorphism can be replaced by $\mathcal{N}_{k}^{(k)} /\left(\mathcal{N}_{k}^{(k)} \cap p \mathcal{M}^{(k)}\right)$. But the latter can be viewed as a $G$-stable subspace $W^{(k)}$ of $V^{(k)}$, and the representation of $G$ on $W^{(k)}$ is therefore equivalent to $\sigma_{k}$. Furthermore, we have seen that $V^{(k)}$ has exactly two irreducible constituents, so the quotient $V^{(k)} / W^{(k)}$ is also irreducible. Since its dimension is $(p+1)-(2 k+1)=$ $p-2 k$, we deduce that the quotient representation is equivalent to $\sigma_{(p-1-2 k) / 2}$. In summary, the representation of $G$ on $W^{(k)}$ and on $V^{(k)} / W^{(k)}$ is equivalent to $\sigma_{k}$ and to $\sigma_{(p-1-2 k) / 2}$ respectively.

To see that $\tau^{(0)} \cong \sigma_{0} \oplus \sigma_{(p-1) / 2}$, we observe that the set of indices $k$ satisfying $1 \leqslant k \leqslant(p-3) / 2$ is stable under $k \mapsto(p-1-2 k) / 2$. It follows that $\sigma_{0}$ and $\sigma_{(p-1) / 2}$ occur as constituents of $V^{(k)}$ if and only if $k=0$. On the other hand, $\sigma_{0}$ and $\sigma_{(p-1) / 2}$ occur not merely as constituents but as subrepresentations of $\tau$ (Proposition 4). It follows that they occur as subrepresentations of $\tau^{(0)}$, whence $\tau^{(0)} \cong \sigma_{0} \oplus \sigma_{(p-1) / 2}$.

Finally, suppose that $1 \leqslant k \leqslant(p-3) / 2$. If $W$ is an irreducible subspace of $V^{(k)}$ then the representation of $G$ on $W$ is equivalent to an irreducible constituent of $\tau^{(k)}$, hence either to $\sigma_{k}$ or to $\sigma_{(p-1-2 k) / 2}$. But if $W \neq W^{(k)}$ then the first possibility is excluded, because $\sigma_{k}$ occurs as a subrepresentation of $\tau$ with multiplicity one (Proposition 4). As for the second possibility, it coincides with the first (and is therefore excluded when $\left.W \neq W^{(k)}\right)$ if $k=(p-1) / 4$. Otherwise it is excluded by Proposition 4 again, because $\sigma_{(p-1-2 k) / 2}$ already occurs as a subrepresentation of $\tau^{((p-1-2 k) / 2)}$, and the spaces $V^{((p-1-2 k) / 2)}$ and $V^{(k)}$ are linearly independent. We conclude that $W^{(k)}$ is the unique irreducible subspace of $V^{(k)}$, and since $V^{(k)}$ has just two irreducible constituents it follows that $V^{(k)} / W^{(k)}$ is the unique irreducible quotient.

\section{THE QUADRATIC RELATIONS}

To move a step closer to $U$ we turn from $M$ to the $\mathbb{Z}[G]$-submodule $Q$ of $M$ defined by the "quadratic relations" of Kubert and Lang. As before, our primary concern is the representation of $G$ on the associated vector space over $\mathbb{F}_{p}$, which is now the space $V^{\prime}=Q / p Q$. 
2.1. Preliminaries. To define $Q$, recall that given $m \in M$ we write $\bar{m}: R \rightarrow \mathbb{F}_{p}$ for the reduction of $m$ modulo $p$. We will also let $N$ denote the $\mathbb{Z}[G]$-submodule of $M$ consisting of all $n$ for which $\bar{n}$ has the form

$$
\bar{n}(r)=a r_{1}^{2}+b r_{1} r_{2}+c r_{2}^{2}
$$

with $a, b, c \in \mathbb{F}_{p}$, where $r=\left(r_{1}, r_{2}\right)$. Since $N$ is a $\mathbb{Z}$-form of the $\mathbb{Z}_{p}[G]$-module previously denoted $\mathcal{N}_{1}$, it might be more logical to denote it $N_{1}$, but for simplicity we omit the subscript (and thereby void our previous convention that $N$ is the subgroup of $G$ corresponding to strictly upper triangular matrices). We define $Q$ to consist of those $m \in M$ such that

$$
\sum_{r \in R} \bar{m}(r) \bar{n}(r)=0
$$

for all $n \in N$.

It is immediate from this description that $Q$ contains $p M$. Thus $M / Q$ is a quotient of the finite-dimensional vector space $V=M / p M$ over $\mathbb{F}_{p}$. In fact since $Q$ is defined by the vanishing of three linearly independent linear forms on $M / p M$ (namely those corresponding to the choices $(a, b, c)=(1,0,0),(0,1,0)$, and $(0,0,1)$ in (9) and (10)) we see that $M / Q$ has dimension three over $\mathbb{F}_{p}$. In particular $Q$ has finite index in $M$, so by the Brauer-Nesbitt theorem, the representation $\tau^{\prime}$ of $G$ on the space $V^{\prime}=Q / p Q$ has the same semisimplification as $\tau$. In other words, Proposition 2 holds with $\tau$ replaced by $\tau^{\prime}$. However Proposition 5 must be modified slightly.

2.2. Homogeneous components, Put $\mathcal{Q}=\mathbb{Z}_{p} \otimes_{\mathbb{Z}} Q$. Then $\mathcal{Q}$ is stable under $e^{(k)}$ (cf. (6), (9), and (10)). Hence

$$
\mathcal{Q}=\oplus_{k=0}^{(p-3) / 2} \mathcal{Q}^{(k)}
$$

with $\mathcal{Q}^{(k)}=e^{(k)} \mathcal{Q}$. Thus putting $V^{\prime(k)}=\mathcal{Q}^{(k)} / p \mathcal{Q}^{(k)}$ we have

$$
V^{\prime}=\oplus_{k=0}^{(p-3) / 2} V^{\prime(k)},
$$

a decomposition of $V^{\prime}$ into $G$-stable subspaces. Let ${\tau^{\prime}}^{(k)}$ denote the representation of $G$ on $V^{(k)}$.

Proposition 6. If $1 \leqslant k \leqslant(p-5) / 2$ then ${\tau^{\prime}}^{(k)}$ has a unique irreducible subrepresentation and a unique irreducible quotient representation, and they are equivalent to $\sigma_{k}$ and $\sigma_{(p-1-2 k) / 2}$ respectively. On the other hand, $\tau^{\prime(0)} \cong \sigma_{0} \oplus \sigma_{(p-1) / 2}$ and $\tau^{\prime((p-3) / 2)} \cong \sigma_{1} \oplus \sigma_{(p-3) / 2}$.

Proof. Suppose first that $k \neq(p-3) / 2$. We claim that $\mathcal{M}^{(k)} \subset \mathcal{Q}$, whence $\mathcal{M}^{(k)}=$ $\mathcal{Q}^{(k)}$. To see this, take $m \in \mathcal{M}^{(k)}$ and $n \in N$, and write

$$
\sum_{r \in R} \bar{m}(r) \bar{n}(r)=\sum_{\ell \in \Lambda} \sum_{r \in R \cap \ell} \bar{m}(r) \bar{n}(r),
$$

where $\Lambda$ is the set of lines through the origin in $\mathbb{F}_{p}^{2}$. For each $\ell \in \Lambda$ choose a vector $r_{\ell} \in R$ spanning $\ell$. Then the inner sum on the right-hand side can be written as a sum over $t \in \mathbb{F}_{p}^{\times}$, with $r=t r_{\ell}$. The homogeneity of $\bar{m}$ and $\bar{n}$ then gives

$$
\sum_{r \in R} \bar{m}(r) \bar{n}(r)=\sum_{\ell \in \Lambda} \bar{m}\left(r_{\ell}\right) \bar{n}\left(r_{\ell}\right) \sum_{t \in \mathbb{F}_{p}^{\times}} t^{2 k+2} .
$$


Since $k \neq(p-3) / 2$ the exponent of $t$ on the right-hand side is $<p-1$ and consequently the inner sum is 0 . Thus $\mathcal{M}^{(k)} \subset \mathcal{Q}$ and $\mathcal{M}^{(k)}=\mathcal{Q}^{(k)}$, as claimed.

It follows that if $k \neq(p-3) / 2$ then ${\tau^{\prime}}^{(k)} \cong \tau^{(k)}$, whence the assertions at hand reduce to those of Proposition 5. To handle the remaining case $k=(p-3) / 2$, we recall that $\tau$ and $\tau^{\prime}$ have isomorphic semisimplifications and are direct sums of their respective homogeneous components $\tau^{(k)}$ and ${\tau^{\prime}}^{(k)}$. Since $\tau^{(k)} \cong \tau^{(k)}$ for $k \neq(p-3) / 2$, we deduce that the semisimplifictions of $\tau^{\prime((p-3) / 2)}$ and $\tau^{((p-3) / 2)}$ are likewise isomorphic. Thus by Proposition $5, \tau^{\prime((p-3) / 2)}$ has exactly two irreducible constituents, namely $\sigma_{(p-3) / 2}$ and $\sigma_{1}$.

Now $\mathcal{M}$ and $\mathcal{Q}$ are also the direct sums of their homogeneous components $\mathcal{M}^{(k)}$ and $\mathcal{Q}^{(k)}$, and we have seen that the vector space $\mathcal{M} / \mathcal{Q}=M / Q$ has dimension three over $\mathbb{F}_{p}$ (cf. (9) and (10)) while $\mathcal{M}^{(k)}=\mathcal{Q}^{(k)}$ for $k \neq(p-3) / 2$. Consequently $\mathcal{M}^{((p-3) / 2)} / \mathcal{Q}^{((p-3) / 2)}$ is also three-dimensional over $\mathbb{F}_{p}$, as is therefore the subspace $Y=p \mathcal{M}^{((p-3) / 2)} / p \mathcal{Q}^{((p-3) / 2)}$ of $V^{\prime((p-3) / 2)}$. Since $\tau^{\prime((p-3) / 2)}$ has just the two irreducible constituents $\sigma_{1}$ and $\sigma_{(p-3) / 2}$ of dimensions 3 and $p-2$ respectively, we deduce that the representation of $G$ on $Y$ is $\sigma_{1}$. Thus $\sigma_{1}$ is a subrepresentation of $\tau^{\prime((p-3) / 2)}$ and $\sigma_{(p-3) / 2}$ is the corresponding quotient representation.

It remains to see that $\sigma_{1}$ is also a quotient representation of $\tau^{\prime((p-3) / 2)}$, whence $\sigma_{(p-3) / 2}$ is a subrepresentation and $\tau^{\prime((p-3) / 2)} \cong \sigma_{1} \oplus \sigma_{(p-3) / 2}$. To this end, consider the bilinear pairing $\prec *, * \succ: Q \times N \rightarrow \mathbb{Z}$ given by

$$
\prec m, n \succ=\frac{1}{p} \sum_{r \in R} m(r) n(r) \quad(m \in Q, n \in N) .
$$

Write $L$ for the $\mathbb{Z}[G]$-submodule of $Q$ consisting of those $m$ such that

$$
\prec m, n \succ \equiv 0 \quad(\bmod p)
$$

for all $n \in N$. Put $\mathcal{L}=\mathbb{Z}_{p} \otimes_{\mathbb{Z}} L$. Then $\mathcal{L}$ is stable under $e^{(k)}$, so putting $\mathcal{L}^{(k)}=e^{(k)} \mathcal{L}$ we have

$$
\mathcal{L}=\oplus_{k=0}^{(p-3) / 2} \mathcal{L}^{(k)} .
$$

We claim that $\mathcal{L}^{((p-3) / 2)}$ contains $p \mathcal{Q}^{((p-3) / 2)}$ and that the quotient space $Z=$ $\mathcal{Q}^{((p-3) / 2)} / \mathcal{L}^{((p-3) / 2)}$ of $V^{\prime(p-3) / 2}$ is of positive dimension $\leqslant 3$. An immediate consequence of the claim is that the representation of $G$ on $Z$ is equivalent to $\sigma_{1}$, so verifying the claim will complete the proof.

It is immediate from the definitions that $L$ contains $p Q$ and hence that $\mathcal{L}$ contains $p \mathcal{Q}$. On the other hand, $\mathcal{L}$ does not contain $p \mathcal{M}$ : for if $m \in M$ is the function taking the value 1 on $( \pm 1,0)$ and 0 elsewhere then $\prec p m, n \succ \not \equiv 0 \bmod p$ for any $n \in N$ satisfying (9) with $a \neq 0$. It follows that for some $k$ with $0 \leqslant k \leqslant(p-3) / 2$ we have $p \mathcal{M}^{(k)} \not \subset \mathcal{L}^{(k)}$. But we have seen that $p \mathcal{Q} \subset \mathcal{L}$ and that $p \mathcal{Q}^{(k)}=p \mathcal{M}^{(k)}$ for $k \neq(p-3) / 2$. Hence $\mathcal{L}^{((p-3) / 2)}$ does not contain $p \mathcal{M}^{((p-3) / 2)}$, and we deduce that $\mathcal{L}^{((p-3) / 2)} / p \mathcal{Q}^{((p-3) / 2)}$ is a subspace of $V^{\prime((p-3) / 2)}$ of positive codimension. On the other hand, the codimension is $\leqslant 3$, because the subspace is defined by the vanishing of three linear forms on $V^{\prime((p-3) / 2)}$ (namely the forms $m+p \mathcal{Q}^{((p-3) / 2} \mapsto \prec m, n \succ$ with $n$ as in $(9)$ and $(a, b, c)=(1,0,0),(0,1,0)$, and $(0,0,1))$. Our claim follows. 


\section{The Kubert-Lang MaP}

Now let $H$ denote the complex upper half-plane. Given a matrix $\tilde{\gamma} \in \operatorname{SL}(2, \mathbb{Z})$, we identify its image $\gamma \in \operatorname{PSL}(2, \mathbb{Z})$ with the fractional linear transformation of $H$ defined by $\gamma$. Thus if $f$ is a function on $H$ and $\tilde{\gamma}$ is the right-hand side of (2) then $f \circ \gamma$ is the function $z \mapsto f((a z+b) /(c z+d))$. As usual, $\Gamma(p)$ denotes the subgroup of $\operatorname{SL}(2, \mathbb{Z})$ defined by the conditions $a \equiv d \equiv 1$ and $b \equiv c \equiv 0 \bmod p$, and the group that we are denoting $U$ - namely the multiplicative group of modular units of level $p$-consists of modular functions for $\Gamma(p)$ which are holomorphic and nowhere vanishing on $H$. We make $U$ into a $\mathbb{Z}[G]$-module via the action

$$
g \cdot f=f \circ \gamma^{-1} \quad(g \in G, f \in U),
$$

where $\gamma \in \operatorname{PSL}(2, \mathbb{Z})$ is any lift of $g$. The resulting representation of $G$ on the vector space $V^{\prime \prime}=U / U^{p}$ over $\mathbb{F}_{p}$ will be denoted $\tau^{\prime \prime}$.

Given $a \in p^{-1} \mathbb{Z}^{2}$ with $a \neq(0,0)$, define the Siegel function $g_{a}$ as in [5], p. 29. For $r \in R$ we put $f_{r}=g_{a}^{12}$, where $a \in p^{-1} \mathbb{Z}^{2}$ is chosen so that $r$ coincides with the residue class of $p a$ modulo $p \mathbb{Z}^{2}$. Since $a$ can be replaced by any element of the coset $a+\mathbb{Z}^{2}$, the function $g_{a}^{12}$ is determined only up to multiplication by a $p$ th root of unity ([5], p. 28, Formula K2), but the coset $f_{r} U^{p}$ is uniquely determined by $r$ because $U^{p}$ contains $\mathbb{C}^{\times}$. Furthermore, if $m \in Q$ then the function

$$
f^{m}:=\prod_{r \in R} f_{r}^{m(r)}
$$

belongs to $U$ ([5], p. 76, Theorem 5.2). Hence the assignment $m+p Q \mapsto f^{m} U^{p}$ defines an $\mathbb{F}_{p}$-linear map $\Phi: V^{\prime} \rightarrow V^{\prime \prime}$.

Proposition 7. The map $\Phi$ is surjective with one-dimensional kernel, and it intertwines $\tau^{\prime}$ with $\tau^{\prime \prime}$.

Proof. The argument echos the proof of Proposition 0 of [7], which in turn merely assembles a number of results from [5]. Let us at least recall the relevant citations: The surjectivity of $\Phi$ follows from [5], p. 83, Theorem 1.3, because $p$ is prime to 12 and thus the map $f U^{p} \mapsto f^{12} U^{p}$ is an automorphism of $U / U^{p}$. That the kernel of $\Phi$ is one-dimensional follows from the surjectivity, because $V^{\prime}$ has dimension $\left(p^{2}-1\right) / 2$ over $\mathbb{F}_{p}$ while $V^{\prime \prime}$ has dimension $\left(p^{2}-3\right) / 2$ ([5], p. 42, Theorem 3.2). Finally, the $G$-equivariance of $\Phi$ follows from [5], p. 27, Formula K1.

Put $V^{\prime \prime(k)}=\Phi\left(V^{\prime(k)}\right)$, so that

$$
V^{\prime \prime}=\oplus_{k=0}^{(p-3) / 2} V^{\prime \prime(k)},
$$

and let $\tau^{\prime \prime(k)}$ denote the representation of $G$ on $V^{\prime \prime(k)}$.

Proposition 8. If $1 \leqslant k \leqslant(p-5) / 2$ then $\tau^{\prime \prime}{ }^{(k)}$ has a unique irreducible subrepresentation and a unique irreducible quotient representation, and they are equivalent to $\sigma_{k}$ and $\sigma_{(p-1-2 k) / 2}$ respectively. On the other hand, $\tau^{\prime \prime(0)} \cong \sigma_{(p-1) / 2}$ and $\tau^{\prime \prime}((p-3) / 2) \cong \sigma_{1} \oplus \sigma_{(p-3) / 2}$.

Proof. Combine Propositions 6 and 7 and observe that $V^{\prime}$ has exactly one $G$-stable subspace of dimension one.

We conclude with some remarks which will lead to a slight reformulation of Proposition 8. Since $p \geqslant 7$, the two direct summands of $\tau^{\prime \prime((p-3) / 2)}$ are inequivalent, 
so there is a unique subspace $W^{\prime \prime((p-3) / 2)}$ of $V^{\prime \prime((p-3) / 2)}$ on which the representation of $G$ is equivalent to $\sigma_{(p-3) / 2}$. We shall refer to the subspace

$$
V_{\mathrm{nc}}^{\prime \prime}=\left(\oplus_{k=0}^{(p-5) / 2} V^{\prime \prime(k)}\right) \oplus W^{\prime \prime((p-3) / 2)}
$$

of $V^{\prime \prime}$ as the noncongruence part of $V^{\prime \prime}$. The congruence part of $V^{\prime \prime}$ is the unique subspace $V_{\mathrm{c}}^{\prime \prime}$ of $V^{\prime \prime((p-3) / 2)}$ on which the representation of $G$ is equivalent to $\sigma_{1}$. Thus

$$
V^{\prime \prime}=V_{\mathrm{nc}}^{\prime \prime} \oplus V_{\mathrm{c}}^{\prime \prime} .
$$

To explain the terminology, let $\mathfrak{K}$ be the field of modular functions for $\Gamma(p)$ and let $\mathfrak{K}^{\text {cc }}$ be the "congruence closure" of $\mathfrak{K}$, in other words the union of the modular function fields for all congruence subgroups of $\operatorname{SL}(2, \mathbb{Z})$. Given any subspace $W$ of $V^{\prime \prime}$, we write $\mathfrak{K}_{W}$ for the Kummer extension of $\mathfrak{K}$ obtained by adjoining the $p$ th roots of all $f \in U$ such that $f U^{p} \in W$. (Note that $\mathfrak{K}^{\times p} \cap U=U^{p}$, so that we can apply Kummer theory with $\mathfrak{K}^{\times} / \mathfrak{K}^{\times p}$ replaced by $U / U^{p}$ : in particular, $\left[\mathfrak{K}_{W}: \mathfrak{K}\right]=|W|$.) We claim that

$$
\mathfrak{K}_{V^{\prime \prime}} \cap \mathfrak{K}^{\mathrm{cc}}=\mathfrak{K}_{V_{\mathrm{c}}^{\prime \prime}} .
$$

Together, (12) and (13) justify the designation "noncongruence part" for $V_{\mathrm{nc}}^{\prime \prime}$.

To prove (13), we recall from the proof of Proposition 6 that the subspace of $V^{\prime((p-3) / 2)}$ on which $G$ acts via $\sigma_{1}$ is $p M / p Q$ (strictly speaking we should identify this subspace as $p \mathcal{M}^{((p-3) / 2)} / p \mathcal{Q}^{((p-3) / 2)}$, not $p M / p Q$, but $\mathcal{M}^{(k)}=\mathcal{Q}^{(k)}$ for $k \neq$ $(p-3) / 2)$. Thus $\Phi(p M / p Q)=V_{\mathrm{c}}^{\prime \prime}$. It follows (see [7], Proposition 2, p. 12) that $\mathfrak{K}_{V_{c}^{\prime \prime}}$ is the field of modular functions for $\Gamma\left(p^{2}\right)$, whence the right-hand side of (13) is contained in the left-hand side. For the reverse inclusion, put

$$
\Gamma=\left\{\gamma \in \mathrm{SL}(2, \mathbb{Z}): f \circ \gamma=f \text { for all } f \in \mathfrak{K}_{V^{\prime \prime}} \cap \mathfrak{K}^{\mathrm{cc}}\right\} .
$$

Then the field of modular functions for $\Gamma$ is the left-hand side of (13). In particular, since the left-hand side of (13) is a subfield of $\mathfrak{K}^{\mathrm{cc}}$ it follows that $\Gamma$ is a congruence subgroup. But the least common multiple of the cusp amplitudes of $\Gamma$ divides $p^{2}$, because the field $\mathfrak{K}_{V^{\prime \prime}}$ is generated over $\mathfrak{K}$ by $p$ th roots of elements of $\mathfrak{K}$. Thus the Wohlfahrt level of $\Gamma$ divides $p^{2}$, and since $\Gamma$ is a congruence subgroup its Wohlfahrt level equals its congruence level by the Fricke-Wohlfahrt theorem [9]:

$$
\Gamma\left(p^{2}\right) \subset \Gamma \text {. }
$$

Taking modular function fields of the two sides of (14) reverses the inclusion and thus gives the inclusion of the left-hand side of (13) in the right-hand side.

Now put $W^{\prime \prime(0)}=V^{\prime \prime(0)}$, and for $1 \leqslant k \leqslant(p-5) / 2$ let $W^{\prime \prime}(k)$ be the unique irreducible subspace of $V^{\prime \prime}(k)$. Then the maximal semisimple subspace of $V_{\mathrm{nc}}^{\prime \prime}$ is $\oplus_{k=0}^{(p-3) / 2} W^{\prime \prime}(k)$, and we obtain:

Proposition 9. The representation of $G$ on the maximal semisimple subspace of $V_{n c}^{\prime \prime}$ is equivalent to $\oplus_{k=1}^{(p-1) / 2} \sigma_{k}$.

\section{REFERENCES}

[1] R. Brauer and C. Nesbitt, On the modular characters of groups, Ann. of Math. 42 (1941), 556 -590 .

[2] B. H. Gross, Representation theory and the cuspidal group of $X(p)$, Duke Math. J. 54 (1987), $67-75$. 
[3] E. Hecke, Über ein Fundamentalproblem aus der Theorie der elliptischen Modulfunktionen, Abh. Math. Sem. Hamb. 6 (1928), 235 - 257 (= Math. Werke \# 28, 525 - 547).

[4] E. Hecke, Über das Verhalten der Integrale 1. Gattung bei Abbildungen, insbesondere in der Theorie der elliptischen Modulfunktionen, Abh. Math. Sem. Hamb. 8 (1930), $271-281$ (= Math. Werke \# 29, 548 - 558).

[5] D. S. Kubert and S. Lang, Modular Units, Springer-Verlag, Grundlehren Math. Wissen. vol. $244,1981$.

[6] Á. Lozano-Robledo, On the surjectivity of Galois representations attached to elliptic curves over number fields, Acta Arith. 117 (2005), 283 - 291.

[7] D. E. Rohrlich, Modular units and the surjectivity of a Galois representation, J. of Number Thy. 107 (2004), 8 - 24.

[8] B. Srinivasan, On the modular characters of the special linear group $S L\left(2, p^{n}\right)$, Proc. London Math. Soc. 14 (1964), $101-114$.

[9] K. Wohlfahrt, An extension of F. Klein's level concept, Ill. J. Math. 8 (1964), 529 - 535.

Department of Mathematics and Statistics, Boston University, Boston, MA 02215

E-mail address: rohrlich@math.bu.edu 